\title{
The role of bideterminants in the representation theory
}

\author{
František Marko \\ Penn State Hazleton, 76 University Drive, Hazleton PA 18202, USA \\ E-mail address: fxm130psu.edu
}

\begin{abstract}
This paper provides a brief survey discussing the role of bideterminants in the representation theory.
\end{abstract}

\section{Introduction}

The purpose of this paper is to explain the role of bideterminants in the representation theory. Our goal is to present a wide scope of applications of bideterminants in the representation theory. In doing so, we will be satisfied with presenting the simplest examples whenever possible; for example instead of discussing reductive algebraic groups we will only talk about a general linear group $G L_{n}$. We want to provide the reader with an introduction to the topics of bideterminants instead of indulging in exhaustive technical details. The reader is advised to consult the references for further details.

In Section 1, we deal with the history and the context in which the bideterminants first emerged, namely in the invariant theory. In Section 2, we give a definition of the bideterminant and a statement of the Straightening Formula. In Section 3, we explain the role of bideterminants in the representation theory of the symmetric group. In Section 4, we review a connection of bideterminants to the representation theory of the general linear group and Schur algebra. In Section 5, we discuss the concept of bideterminants related to the representation theory of the general linear supergroup and Schur superalgebra. In Section 6, we consider the role of bideterminants in quantum groups.

2000 Mathematics Subject Classification. 17A70, 17B60, 20 B30.

Key words: bideterminants, general linear group, general linear supergroup, Schur superalgebras, semistandard tableaux, modular reduction. 


\section{Emergence of bideterminants in the invariant theory}

We will start with a discussion of the invariant theory, since it is where the bideterminants first appeared. Determinantal expressions first appeared as primary covariants in the work of Deruyts, while the concepts of a standard tableaux and polarization operators first appeared in the work of Clebsch.

The main results of the invariant theory over a field of characteristic zero were the Gordan-Capelli expansion and the Theorem of E. Pascal.

The work of A. Young on substitutional analysis laid the groundwork for the theory of bideterminants. The concept of a tableaux reappeared in his work [42]. In [43] he reintroduced standard tableaux and proved the first version of the straightening algorithm.

The precursor of bideterminants first appeared in the work of Hodge [22], who considered power products corresponding to tableaux. He proved a certain reduction theorem that extended the original ideas of Young.

The work of Hodge was used by Igusa in [25], where he derived the first results about invariants over a field of arbitrary characteristic. Namely, he proved the following First Fundamental Theorem of vector invariants for $G L_{n}$.

Theorem 1.1. If $F(X)$ is a polynomial in $X_{i j}$ with coefficients in a field $K$ which is invariant under unimodular substitutions $X_{i j}^{\prime}=\sum_{k=0}^{n} a_{i k} X_{k j}$, then $F(X)$ can be expressed as a polynomial in the

$$
P_{i_{0} \ldots i_{k}}=\left|\begin{array}{cccc}
X_{0, i_{0}} & X_{0, i_{1}} & \ldots & X_{0, i_{n}} \\
X_{1, i_{0}} & X_{1, i_{1}} & \ldots & X_{1, i_{n}} \\
\ldots & \ldots & \ldots & \ldots \\
X_{r, i_{0}} & X_{r, i_{1}} & \ldots & X_{r, i_{n}}
\end{array}\right|
$$

with coefficients in $K$.

The breakthrough in the theory of invariants for the field of an arbitrary characteristic came in the work [18] of Doubilet, Rota and Stein. They have considered a pair $\left(T, T^{\prime}\right)$ of Young tableaux, called a bitableuax, defined a bideterminant corresponding to such a bitableaux and proved the Straightening Formula for bideterminants. The Straightening Formula holds over the ring of integers and its proof is based on elementary linear algebra and combinatorics.

In [12], an algorithm for computing the straightening coefficients using Capelli operators was shown and the Second Fundamental Theorem of vector invariants was proved. This theorem describes the kernel of the Pascal 
homomorphism as a subspace spanned by standard bideterminants of specific shapes. The following statement, which is Theorem 3.4 of [9], describes relations between invariants for $G L_{n}$.

Theorem 1.2. The ideal of relations between vector invariants is generated by $(n+1) \times(n+1)$ determinants.

The above results were further generalized in [21] using arguments involving letterplace superalgebras, divided powers, Laplace pairings and fourfold algebras. A recent paper [3] is an elementary introduction to the characteristic zero theory of letterplace superalgebras and it provides an extensive bibliography on this subject.

Invariants of other simple algebraic groups were also considered in the literature.

\section{Bideterminants and straightening formula}

Fix an integer $m$ and denote by $\lambda=\left(\lambda_{1}, \ldots, \lambda_{n}\right)$ a partition of $r$. The diagram $[\lambda]$ of the shape $\lambda$ is a set of numbers $(a, b)$, where $b=1, \ldots, \lambda_{a}$ for $a=1, \ldots n$. A tableaux $T$ is a map that assigns to each element $(a, b) \in[\lambda]$ a number in the set $\{1, \ldots, m\}$. The partition $\lambda$ is called the shape of the tableaux $T$. A tableaux $T$ is called basic if map given by $T$ is injective. Fix a bijection between the set $\{1, \ldots, m\}$ and $[\lambda]$ by listing elements of the diagram $[\lambda]$ by consecutive rows from left to right. Then the tableaux $T$ corresponds to a certain multi-index $w(T)=i=\left(i_{1}, \ldots, i_{m}\right)$ and we will write $T_{i}$ instead of $T$. Denote the entry of the tableaux $T_{i}$ at the position $(a, b)$ by $i_{a, b}$.

Example 2.1. Diagram $T$ of shape $\lambda=(5,3,3,1)$ for $G L(5)$ is

$$
\begin{array}{lllll}
\circ & 0 & 0 & 0 & 0 \\
\circ & 0 & 0 & & \\
\circ & 0 & 0 & & \\
0 & & & &
\end{array}
$$

and the tableaux $T_{i}$ of shape $\lambda$ corresponding to multi-index $i$ is

$\begin{array}{ccccc}i_{1} & i_{2} & i_{3} & i_{4} & i_{5} \\ i_{6} & i_{7} & i_{8} & & \\ i_{9} & i_{10} & i_{11} & & \\ i_{12} & & & & \end{array}$.

The symmetric group $\Sigma_{r}$ acts on the tableaux by permuting its entries. If $\sigma \in \Sigma_{r}$ acts on $T_{i}$, then the resulting tableaux is denoted by $T_{i \sigma}$. Denote the Young subgroup of $\Sigma_{r}$ that consists of all permutations that keeps all columns (respectively rows) of $T$ stable by $C(T)$ (respectively $R(T)$ ) and 
call such permutations column permutations (respectively row permutations) of $T$.

A tableaux $T_{i}$ is called standard if its entries are weakly increasing from left to right in each row and its entries are strongly increasing down each column. More precisely, it is required that $i_{a, b} \leq i_{a, c}$ for $b<c$ and $i_{a, b}<i_{d, b}$ for $a<d$. The tableaux $T_{\ell}$ such that all entries in its $a$-th rows are equal to $a$ will be called canonical. Clearly, $T_{\ell}$ is always a standard tableaux.

Example 2.2. If $m=5, \lambda=(5,3,3,1)$ and $i=(1,2,2,4,5,2,3,3,3$, $4,5,5)$, then $T_{\ell}$ and $T_{i}$ can be visualized as

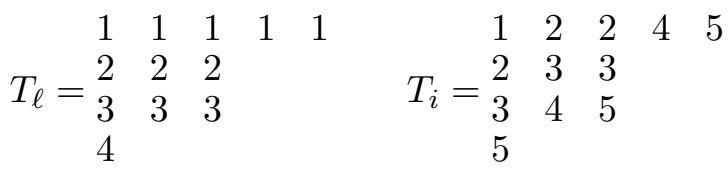

and $T_{i}$ is standard.

For a tableau $T$ define its content $\operatorname{cont}(T)$ to be an $m$-tuple that describes the number of occurrences of elements $1, \ldots, m$ in $T$. Then $\operatorname{cont}\left(T_{\ell}\right)=\lambda$ and $\operatorname{cont}\left(T_{i}\right)=(1,3,3,2,2)$ for $T_{i}$ as above.

A bitableaux is a pair of tableaux $\left(T_{i} \mid T_{j}\right)$ of the same shape. Denote a pairing of multi-indices $i, j$ by $(i \mid j)=\left(i_{1} \mid j_{1}\right) \ldots\left(i_{r} \mid j_{r}\right)$. For simplicity denote $(i \mid j)$ by $c_{i, j}=c_{i_{1}, j_{1}} \ldots c_{i_{r}, j_{r}}$. Then to a bitableaux $\left(T_{i} \mid T_{j}\right)$ we assign a bideterminant $\left(T_{i}: T_{j}\right)$ defined as

$$
\left(T_{i}: T_{j}\right)=\sum_{\sigma \in C(T)} \operatorname{sgn}(\sigma)(i \mid j \sigma)=\sum_{\sigma \in C(T)} \operatorname{sgn}(\sigma) c_{i, j \sigma} .
$$

To illustrate this definition, we write $\left(T_{\ell}: T_{i}\right)$, for $T_{\ell}, T_{i}$ as in the above example, as the following product of determinants

$$
\left|\begin{array}{llll}
c_{1,1} & c_{1,2} & c_{1,3} & c_{1,5} \\
c_{2,1} & c_{2,2} & c_{2,3} & c_{2,5} \\
c_{3,1} & c_{3,2} & c_{3,3} & c_{3,5} \\
c_{4,1} & c_{4,2} & c_{4,3} & c_{4,5}
\end{array}\right| \begin{array}{ccc}
c_{1,2} & c_{1,3} & c_{1,4} \\
c_{2,2} & c_{2,3} & c_{2,4} \\
c_{3,2} & c_{3,3} & c_{3,4}
\end{array}\left|\begin{array}{ccc}
c_{1,2} & c_{1,3} & c_{1,5} \\
c_{2,2} & c_{2,3} & c_{2,5} \\
c_{3,2} & c_{3,3} & c_{3,5}
\end{array}\right|\left|c_{14}\right|\left|c_{15}\right| .
$$

The main tool in the proof of the straightening formula is the Laplace duality (See Theorem 2.5.1 of [33]).

Proposition 2.3. Let $\lambda$ and $\mu$ be partitions of $r, T$ be a tableaux of shape $\lambda$ and $S$ be a tableaux of shape $\mu$. Let $X$ be a transversal of left cosets of $C(T) \cap C(S)$ in $C(S)$, and $Y$ be a transversal of right cosets of $C(T) \cap C(S)$ 
in $C(T)$. Then for every $i, j$,

$$
\sum_{\pi \in X} \operatorname{sgn}(\pi)\left(T_{i \pi}: T_{j}\right)=\sum_{\sigma \in Y} \operatorname{sgn}(\sigma)\left(S_{i}: S_{j \sigma^{-1}}\right) .
$$

Before we can formulate the statement of the straightening formula, we need to define the following linear orders. First we define a linear order $\leq$ on partitions by ordering them lexicographically. Further, define a linear order $\leq$ on tableaux $T$ of the same shape $\lambda$ by lexicographic ordering on multi-indices $w(T)$. For tableaux from Example 2.2 we have $T_{\ell}<T_{i}$. A linear order on the set of all tableaux is given by $T \leq S$ if and only if the shape of $T$ is smaller than the shape of $S$, or the shapes of $T$ and $S$ are the same and $w(T) \geq w(S)$. This induces a partial order on bitableaux $(S \mid T)$ defined by $(S \mid T) \leq\left(S^{\prime} \mid T^{\prime}\right)$ if and only if $S \leq S^{\prime}$ and $T \leq T^{\prime}$.

For the following formulation of the straightening formula see Theorem 8.2 of $[3]$.

Theorem 2.4. (Straightening formula). The bideterminants $(S: T)$, where $S, T$ are standard tableaux of the same shape, form a basis of the algebra $A=K\left[c_{i j}\right]$. If $S$ and $T$ are arbitrary tableaux of the shape $\lambda$, then

$$
(S: T)=\sum_{i} a_{i}\left(S_{i}: T_{i}\right),
$$

where the tableaux $S_{i}$ and $T_{i}$ are standard and $(S \mid T) \leq\left(S_{i} \mid T_{i}\right)$, cont $\left(S_{i}\right)=$ $\operatorname{cont}(S), \operatorname{cont}\left(T_{i}\right)=\operatorname{cont}(T)$, and all coefficients $a_{i}$ are integers for every index $i$.

\section{Example 2.5.}

$$
\left(\begin{array}{l|l}
2 & 1 \\
1 & 2
\end{array}\right)=\left(\begin{array}{l|l}
1 & 1 \\
2 & 2
\end{array}\right)-\left(\begin{array}{ll|ll}
1 & 2 & 1 & 2
\end{array}\right)
$$

There is an algorithm that determines the coefficients $a_{i}$ from the straightening formula at bideterminant $\left(S_{i} \mid T_{i}\right)$ of shape $\lambda$ using Capelli operators or Desarmenien matrix and Schur coefficients - see [11], [12] and [1].

For connection to determinantal varieties see [10].

Bideterminants related to other algebraic groups were also considered. Within the context of symplectic groups see papers [29], [8], [16], [41] and [39]. For bideterminants for the orthogonal group see [6] and [30].

\section{Representation theory of the symmetric group}

The representation theory of the symmetric group $\Sigma_{r}$ over the field $\mathbb{C}$ of complex numbers was developed by Frobenius. The connection to the 
representation theory of the general linear group was discovered by Schur. Afterwards, the understanding of representations of the symmetric group was significantly advanced by A. Young.

Modular representation theory of the symmetric group is not completely understood but notable results in this directions were obtained by James.

Instead of representations of $\Sigma_{n}$ we will talk about representations (or modules) over the group algebra $K \Sigma_{n}$. If $K$ is a field of characteristic zero, then by Maschke Theorem every representation of $\Sigma_{n}$ is completely reducible. Therefore we need to describe irreducible representations only.

Let $\lambda=\left(\lambda_{1}, \ldots, \lambda_{n}\right)$ be a partition of $r$ and $T_{i}, T_{j}$ be tableaux of shape $\lambda$. Tableaux $T_{i}$ and $T_{j}$ are row equivalent if $i=j \sigma$ for $\sigma \in R(T)$. An equivalence class $\{T\}$ of tableaux of shape $\lambda$ is called a $\lambda$-tabloid. The tabloid $\{T\}$ is called basic if $T$ is basic.

Since we have already defined the action of $\Sigma_{r}$ on $T_{i}$ on the right but it is customary to denote the action of $\Sigma_{r}$ on the left, we define $\sigma T_{i}=T_{i \sigma^{-1}}$ and $\sigma\left\{T_{i}\right\}=\left\{T_{i \sigma^{-1}}\right\}$ for $\sigma \in \Sigma_{r}$.

Define the element $\kappa(T)$ of $K \Sigma_{r}$ by the formula

$$
\kappa(T)=\sum_{\pi \in C(T)} \operatorname{sgn}(\pi) \pi
$$

and denote by $e(T)$ the associated polytabloid $\kappa(T)\{T\}$. The polytabloid $e(T)$ is called basic if $T$ is basic.

Example 3.1. Let $T=\begin{array}{llll}1 & 2 & 3 \\ 4 & 5\end{array}$. If we depict the tabloid $\{T\}$ as \begin{tabular}{lll}
\hline 1 & 2 & 3 \\
\hline 4 & 5 &
\end{tabular} and analogously for other tabloids, then

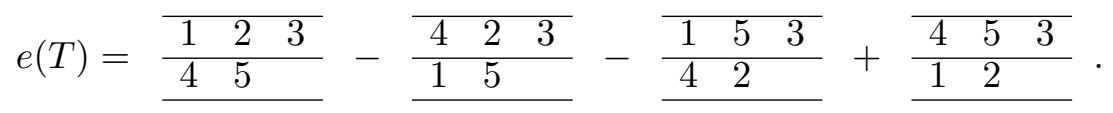

Denote by $M^{\lambda}$ the permutation module corresponding to $\lambda$, spanned by the complete list of basic $\lambda$-tabloids, and by $S^{\lambda}$ the Specht module spanned by all basic polytabloids $e(T)$ of shape $\lambda$.

A partition $\lambda$ is called $p$-regular, if there is no index $i$ such that $\lambda_{i}=$ $\lambda_{i+1}=\ldots=\lambda_{i+p}>0$.

Now we are ready to formulate the following crucial result providing a description of irreducible $K \Sigma_{r}$ modules (See Theorem 11.5 of [26] or [37]).

Theorem 3.2. If the characteristic of $K$ is zero, then the Specht modules $S^{\lambda}$ for arbitrary partitions $\lambda$ of $r$ form a complete set of irreducible $K \Sigma_{r}$ modules. 
If the characteristic of $K$ is $p>0$, then a complete set $D^{\lambda}$ of irreducible $K \Sigma_{r}$-modules is indexed by $p$-regular partitions $\lambda$ of $r$. Moreover, every $D^{\lambda}$ is a factormodule of the Specht module $S^{\lambda}$.

The following theorem is an analogy of Theorem 2.4 in the context of symmetric groups. We will explain the connection of this theorem to bideterminants in the following section.

Theorem 3.3. The set of all $e(T)$, where $T$ is a basic standard tableau of the shape $\lambda$ is a basis for $S^{\lambda}$.

An inductive proof of Theorem 3.3 uses the following column dominance order on basic tabloids and Garnir relations.

For $a=1, \ldots, m$ denote by $c_{a, b}(i)$ the number of entries in the first $a$ rows of the tableau $T_{i}$ that are smaller than or equal to $b$. Denote by $c(i)$ the tuple $\left(c_{a, b}(i) \mid a=1, \ldots, k\right)$ and impose the lexicographic order $\prec$ on tuples $c(i)$, by comparing entries with respect to $a$ first, followed by $b$ second, etc. This order induces a linear order on tabloids $\{T\}$ of the shape $\lambda$. Analogously to $\prec$, we define a column linear order $\prec_{c}$ on tableaux $T$ of the shape $\lambda$ by replacing rows by columns of $T$ in the definition above.

The Garnir relations provide dependence relationships between basic tabloids which allows a replacement of a basic tabloid with an inversion in one of its columns by an integral linear combination of basic tabloids that are smaller with respect to the order $\prec_{c}$.

The Garnir relations, that are related to the Laplace duality, are defined as follows. Let $X$ be a subset of the $i$-th column of $T$, and $Y$ be a subset of the $(i+1)$-th column of $T$. Let $\sigma_{1}, \ldots, \sigma_{k}$ be the coset representative for $\Sigma_{X} \times \Sigma_{Y}$ in $\Sigma_{X \cup Y}$ and let $G_{X, Y}=\sum_{j=1}^{k} \operatorname{sgn}\left(\sigma_{j}\right) \sigma_{j}$.

Proposition 3.4. If the cardinality of $X \cup Y$ exceeds the length of the $i$-th column of $T$, then $e(T) G_{X, Y}=0$.

The well-known Schur-Weyl duality relates the representation theories of the symmetric group $\Sigma_{r}$ and the general linear group $G L_{n}$ as follows. Let $G L_{n}$ act on $E=K^{n}$ naturally and on $E^{\otimes r}$ diagonally; and let $\Sigma_{r}$ act on $E^{\otimes r}$ by place permutations. If $n \geq r$, then $\operatorname{Hom}_{K \Sigma_{r}}\left(E^{\otimes r}\right) \cong S(n, r)$ and $\operatorname{Hom}_{S(n, r)}\left(E^{\otimes r}\right) \cong K \Sigma_{r}$, where $S(n, r)$ is the Schur algebra generated by the image of the above $G L_{n}$-action.

The ideas behind Theorem 3.3 were extended to the description of a basis $\operatorname{Hom}_{\Sigma_{r}}\left(S^{\lambda}, M^{\mu}\right)$ and connected to the representation theory of the general linear group by Carter and Lusztig in [5]. The main difference is that they considered all tableaux $T$, not only the basic ones as in the previous section. 
Let $\mu=\left(\mu_{1}, \ldots, \mu_{m}\right)$ be a decomposition of $r$, that is $\mu_{1}+\ldots \mu_{m}=r$. For any partition $\lambda$, the vector space spanned by all tableaux $T$ of shape $\lambda$ and content $\mu$ is isomorphic to $M^{\mu}$, see Proposition 2.9.2 of [37]. Using this isomorphism, we define a morphism $\theta_{T} \in \operatorname{Hom}_{K \Sigma_{r}}\left(M^{\lambda}, N^{\mu}\right)$ by sending a fixed basic tableaux to a sum of all tableaux that are row equivalent to $T$. Let $\bar{\theta}_{T}$ be a restriction of $\theta_{T}$ to $S^{\lambda}$.

Theorem 3.5. The set of morphisms $\bar{\theta}_{T}$ for semistandard tableaux $T$ of shape $\lambda$ and content $\mu$ is a basis of $\operatorname{Hom}_{K \Sigma_{r}}\left(S^{\lambda}, M^{\mu}\right)$.

The proof of this theorem can be found in [26], [28] or [37].

\section{Representation theory of the general linear group and Schur algebra}

4.1. char $\mathbf{K}=\mathbf{0}$. If $\operatorname{char} K=0$, then every module over $G L_{n}$ and $S(n, r)$ is completely reducible and the highest weights of irreducible modules correspond to dominant weights $\lambda$ with no more than $n$ non-zero components. The irreducible $S(n, r)$-modules $D_{\lambda}$ will be described below in terms of bideterminants.

Let $\lambda=\left(\lambda_{1} \geq \lambda_{2} \geq \ldots \lambda_{n}\right)$ be a dominant weight of $G L_{n}$. Define $D_{\lambda}$ to be the vector space spanned by all bideterminants $\left(T_{\ell}: T_{i}\right)$ of shape $\lambda$. It is verified that $D_{\lambda}$ is an $S(n, r)$-module. The expression $c_{\ell, i}=c_{\ell, j}$ if and only if $\ell \sigma=\ell$ and $i \sigma=j$ for some $\sigma \in \Sigma_{r}$. Therefore $c_{\ell, i}=c_{\ell, j}$ if and only if $T_{j}$ is obtained from $T_{i}$ by some $\sigma \in R(T)$. Therefore the expressions $c_{\ell, j}$ are in one-to-one correspondence to tabloids $\left\{T_{j}\right\}$. Consequently, a lot of combinatorial techniques from the representation theory of the symmetric group $\Sigma_{r}$ are at our disposal.

James (Theorem 26. 6 of [26]) investigated a $G L_{n}$-module isomorphic to $D_{\lambda}$ and Green (Theorem 4.8e of [19]) interpreted James's results in the following way.

Let $T_{n}$ and $U_{n}^{-}$be subgroups of $G L_{n}$ consisting of diagonal and lower triangular unipotent matrices, respectively, and $\chi^{\lambda}$ be the character of $T_{n}$ corresponding to $\lambda$. Define $B_{n}^{-}=T_{n} U_{n}^{-}$to be the Borel subgroup of $G L_{n}$ and the $K B_{n}^{-}$-module $K_{\lambda}=K$ by conditions $t .1=\chi^{\lambda}(t)$ and $u .1=u$ for $t \in T_{n}$ and $u \in U_{n}^{-}$. Theorem 3.2.6. of [33] states the following.

Theorem 4.1. The module $D_{\lambda}$ is isomorphic to the induced module $\operatorname{Ind}_{B_{n}^{-}}^{G L_{n}}\left(K_{\lambda}\right)=H^{0}(\lambda)$.

The proof of the following theorem in the setting of the general linear group that can be found in [19] or [33] uses Carter-Lustig lemma or analogues of Garnir relations as adapted by Peel [40]. 
Theorem 4.2. The $S(n, r)$-module $D_{\lambda}$ is irreducible and its basis consists of all bideterminants $\left(T_{\ell}: T_{i}\right)$, where $T_{i}$ are standard tableaux of shape $\lambda$. Additionally, every bideterminant $\left(T_{\ell}: T\right)$ is an integral linear combination of $\left(T_{\ell}: T_{i}\right)$, where $T_{i}$ are semistandard tableaux.

The second part of the last theorem opens a door for the modular reduction as outlined in [5] or [19].

4.2. Modular reduction. We will outline the general theory first. Assume that $\operatorname{char} K=p>0$. Let $A$ be an algebra over $\mathbb{Q}$ and $A_{\mathbb{Z}}$ be a lattice in $A_{\mathbb{Q}}$ which is closed under multiplication. Then the $K$-algebra $A_{K}=A_{\mathbb{Z}} \otimes_{\mathbb{Z}} K$ is a modular reduction of $A_{\mathbb{Q}}$ to the field $K$. A $\mathbb{Z}$-form of the $A_{\mathbb{Q}}$-module $M$ is a lattice $M_{\mathbb{Z}}$ inside $M$ such that $M_{\mathbb{Z}} \otimes_{\mathbb{Q}} Q \cong M$ and $M_{\mathbb{Z}}$ is closed under the action of elements from $A_{\mathbb{Z}}$. Then $M_{\mathbb{Z}} \otimes_{\mathbb{Z}} K=M_{K}$ is called a modular reduction of the module $M$ to the field $K$. The structure of the module $M_{K}$ depends on the choice of the $\mathbb{Z}$-form $M_{\mathbb{Z}}$ but the multiplicities $\left[M_{K}: L_{K}(\lambda)\right]$ of simple modules $L_{K}(\lambda)$ in each modular reduction $M_{K}$ are the same.

There is a natural $\mathbb{Z}$-form $S_{\mathbb{Z}}(n, r)$ of the algebra $S_{\mathbb{Q}}(n, r)$, where the basis elements consists of some elements $\xi_{i j}$ dual to polynomials $c_{i j}$. Additionally, the action of $S_{\mathbb{Z}}(n, r)$ on bideterminants $\left(T_{\ell}: T_{i}\right)$ produces a $\mathbb{Z}$-linear combination of $\left(T_{\ell}: T_{j}\right)$. Therefore the $\mathbb{Z}$-span $D_{\lambda, \mathbb{Z}}$ of bideterminants $\left(T_{\ell}: T_{i}\right)$ is a $\mathbb{Z}$-form of the module $D_{\lambda, \mathbb{Q}}$.

After the modular reduction of $D_{\lambda, \mathbb{Q}}$, the $S_{\mathbb{Z}}(n, r) \otimes K$ - modules $D_{\lambda, \mathbb{Z}} \otimes K$ do not remain irreducible. The multiplicities $\left[D_{\lambda, \mathbb{Z}} \otimes K: L_{K}(\mu)\right]$, where $L(\mu)$ are simple $S_{\mathbb{Z}}(n, r) \otimes K$-modules, are called decomposition numbers. For results on decomposition numbers, see [27] or [33].

There is a natural non-singular bilinear form between $S(n, r)$ and the set $A(n, r)$ of polynomials in $c_{i, j}$ of degree $r$. Under this pairing, the dual of the Schur module $D_{\lambda}$ is the Weyl module $W_{\lambda}$ investigated in [5]. (Also see [19] or [33]).

Each Schur module $D_{\lambda}$ has a unique minimal submodule $L(\lambda)$, and each Weyl module $W_{\lambda}$ has a unique minimal factormodule $F(\lambda)$. Using these properties, it is possible to prove that even if $\operatorname{char} K=p>0$, the highest weights of irreducible modules correspond to dominant weights $\lambda$ with no more than $n$ non-zero components, exactly as was the case when $\operatorname{char} K=$ 0 .

Cline, Parshall and Scott defined the concepts of the highest weight category and quasi-hereditary algebra in [7]. These concepts describe deep structural properties of algebras and their module categories. The following theorem was first proved by Donkin in [15]. (See also [19] and [33]). 
Theorem 4.3. The algebra $S_{K}(n, r)$ is a quasi-hereditary algebra.

In [20], Green introduced the concept of the codeterminant. Using a connection between bideterminants and codeterminants given through the Desarmenien matrix he gave a combinatorial proof of the above theorem. He used codeterminants to describe a basis of the Schur algebra and to give a filtration of the Schur algebra, with factors isomorphic to Weyl modules $W_{\lambda}$. Relating the standard basis of $W_{\lambda}$ given by Carter and Lusztig in [5] he exhibited another basis of $W_{\lambda}$ given by codeterminants.

The reader might wonder if there is any application of a more general straightening formula for bideterminants formulated in Theorem 2.4. With the help of that theorem we get a very explicit description of the $S(n, r)$ module $A(n, r)$. However, to understand the structure of $S(n, r)$ we need to use different devices like the above mentioned codeterminants and their connection to bideterminants. For details see [20] and [33].

\section{Representation theory of the general linear supergroup and Schur superalgebra}

Since the general linear supergroup and Schur superalgebras are perhaps not known to the reader, we start with the outline of their definitions. For details see [4] or [44].

Assume that $K$ is a field of characteristic $p \neq 2$. Fix an alphabet consisting of $m$ even symbols $1, \ldots, m$ and $n$ odd symbols $m+1, \ldots, m+n$ and declare the parity $\left|c_{i j}\right|$ of $c_{i j}$ as the sum of parities of $i$ and $j$. Elements $c_{i j}, 1 \leq i, j \leq m+n$, are subject to supercommutativity relation $c_{i j} c_{k l}=(-1)^{\left|c_{i j}\right|\left|c_{k l}\right|} c_{k l} c_{i j}$.

Denote by $A(m \mid n)$ a commutative superalgebra freely generated by elements $c_{i j}, 1 \leq i, j \leq m+n . A(m \mid n)$ is a superbialgebra with respect to the comultiplication $\Delta\left(c_{i j}\right)=\sum_{1 \leq k \leq m+n} c_{i k} \otimes c_{k j}$ and counit $\epsilon\left(c_{i j}\right)=\delta_{i j}$. The algebra $A(m \mid n)=\oplus_{r \geq 0} A(m \mid n, r)$ has obvious grading where every homogeneous component $A(m \mid n, r)$ is a supercoalgebra. Its dual $S(m \mid n, r)=$ $A(m \mid n, r)^{*}$ is a Schur superalgebra.

Write a generic matrix $C=\left(c_{i j}\right)_{1 \leq i, j \leq m+n}$ as a block matrix $\left(\begin{array}{ll}C_{00} & C_{01} \\ C_{10} & C_{11}\end{array}\right)$, where entries of $C_{00}$ and $C_{11}$ are even and entries of $C_{01}$ and $C_{10}$ are odd. The localization of $A(m \mid n)$ by elements $\operatorname{det}\left(C_{00}\right)$ and $\operatorname{det}\left(C_{11}\right)$ is the coordinate superalgebra $K[G]$ of the general linear supergroup $G=G L(m \mid n)$. 
The category of modules over $S(m \mid n, r)$ is isomorphic to the category of polynomial representations of degree $r$ over the general linear supergroup $G L(m \mid n)$.

Assume now that char $K=0$. The algebra $S(m \mid n, r)$ was investigated by Sergeev [38], and Berele and Regev in [2]. Berele and Regev defined a *-action of the symmetric group $\Sigma_{r}$ on $E^{\otimes r}$, where the natural module $E$ is an analogue of the module considered for Schur algebras. The $*$-action differs from the previous action of $\Sigma_{r}$ defined by place permutations only by a factor of \pm 1 .

Berele and Regev proved that $S(m \mid n, r)$ is a semisimple algebra and its simple modules $D_{\lambda}$ correspond to $(m \mid n)$-hook partition $\lambda=\left(\lambda_{1} \geq \ldots \geq \lambda_{k}\right)$ for which $\lambda_{m+1} \leq n$.

Muir in [35] exhibited the basis of $D_{\lambda}$ in terms of expressions of determinantal type that he also called bideterminants. In order to describe them, we need more definitions.

A tableaux $T$ of the shape $\lambda$ is a map from the set $\{1, \ldots, r\}$ to $\{1, \ldots, m+n\}$. We will split the partition $\lambda$ and the tableau $T$ into two parts. Define $e_{e} \lambda=\left(\lambda_{1}, \ldots, \lambda_{m}\right)$ and denote by $e_{e} T$ the tableau consisting of the first $m$ rows of $T$. The remaining entries of $\lambda$ (if any) form a partition ${ }_{o} \lambda=\left(\lambda_{m+1}, \ldots, \lambda_{k}\right)$ and the remaining rows (if any) of $T$ form a tableau denoted by ${ }_{o} T$. Denote $\left(\lambda_{1}^{\prime}, \ldots, \lambda_{k^{\prime}}^{\prime}\right)$, the conjugate of the partition ${ }_{o} \lambda$, by ${ }_{o} \lambda^{\prime}$. Denote by $\ell$ a unique element corresponding to the canonical tableau $T_{\ell}$ that satisfies $T_{\ell}(i, j)=i$ for $i \leq m$ and $T_{\ell}(i, j)=m+j$ for $i>m$.

A tableau $T_{i}$ is called $(m \mid n)$-semistandard if:

i) Entries in each row of $T_{i}$ are weakly increasing from left to right and entries in the same column of $T_{i}$ are weakly increasing from top to bottom,

ii) Entries from the set $\{1, \ldots, m\}$ in the same column of $T_{i}$ are strongly increasing, and

iii) Entries from the set $\{m+1, \ldots, m+n\}$ in the same row of $T_{i}$ are strongly increasing

Example 5.1. The diagram of shape $\lambda=(5,3,3,1)$ for $S(1 \mid 4)$ can be

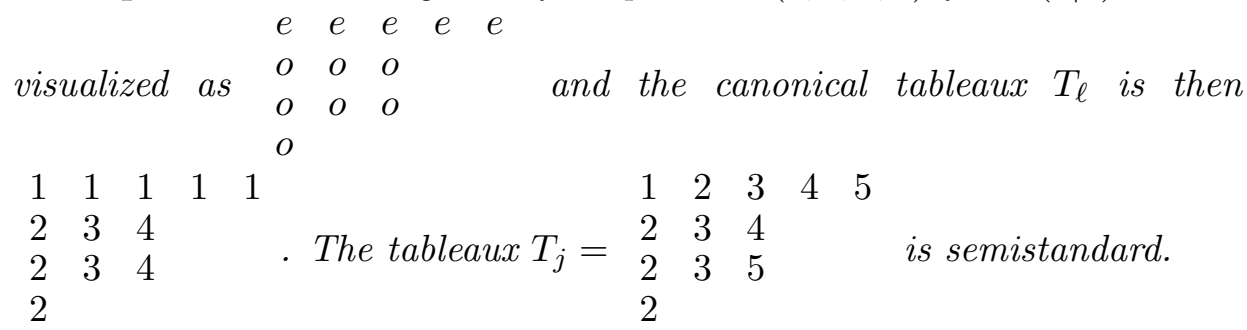


In order to define bideterminants for $S(m \mid n, r)$, for technical reasons, we will replace elements $c_{i j}$ by elements $\xi_{i, j}$ that differ from $c_{i, j}$ only by a factor \pm 1 . Then $\xi_{i, j * \sigma}=( \pm 1) \xi_{i, j \sigma}$ for appropriate value of \pm 1 . For tableaux $T_{\ell}$, $T_{i}$ of shape $\lambda$ define the bideterminant

$$
\left(T_{\ell}: T_{j}\right)=\sum_{\rho \in R(o T)} \sum_{\kappa \in C(T)} \operatorname{sgn}(\kappa) \chi_{\ell, j * \kappa * \rho} .
$$

Denote by $D_{\lambda}$ the $K$-span of bideterminants $\left(T_{\ell}: T_{j}\right)$ of shape $\lambda$. The following theorem is proven in [35].

Theorem 5.2. Let $\lambda$ be a $(m \mid n)$-hook partition of $r$. Then $D_{\lambda}$ is an irreducible $S(m \mid n, r)$-module. The $K$-basis of $D_{\lambda}$ consists of bideterminants $\left(T_{\ell}: T_{i}\right)$ for semistandard $T_{i}$.

The $\mathbb{Z}$-span of bideterminants $\left(T_{\ell}: T_{j}\right)$ is not a $\mathbb{Z}$-form of $D_{\lambda}$. A $\mathbb{Z}$-form of $D_{\lambda}$ is obtained by $\mathbb{Z}$-span of modified bideterminants defined as follows.

Denote by $n_{j, a, b}$ the number of entries in the $a$ column of $T_{j}$ that are equal to $b$, and by $n_{j}$ the product $\prod_{a=1}^{\lambda_{1}} \prod_{b=m+1}^{m+n} n_{j, a, b}$ !. The modified bideterminants $\left[T_{\ell}: T_{j}\right]$ are given as $\left[T_{\ell}: T_{j}\right]=\frac{1}{n_{j}}\left(T_{\ell}: T_{j}\right)$. The following theorem is formulated in [32].

Theorem 5.3. The $\mathbb{Z}$-span of modified bideterminants $\left[T_{\ell}: T_{i}\right]$ is a $\mathbb{Z}$ form of the simple $S(m \mid n, r)$-module $D_{\lambda, \mathbb{Q}}$. Every modified bideterminant $\left[T_{\ell}: T_{j}\right]$ is a $\mathbb{Z}$-linear combination of modified bideterminants $\left[T_{\ell}: T_{i}\right]$ for $T_{i}$ semistandard.

The proof uses computations with superderivations, a concept of an even row tabloid, an analogue of Garnir relations and a super version of Clausen order.

From the structural point of view, the algebras $S(m \mid n, r)$ are quasihereditary if and only if they are semisimple. A complete description of this situation is given in [31].

Finally, we will describe highest weights of simple modules in the modular case. This description is due to Brundan and Kujawa [4].

Recall that the $p$-rim of the diagram of $\lambda$ is a subset of the rim consisting of union of the $p$ segments. To form $p$-segments we read along the rim from left to right until we reach the $p$-th node. To get the next $p$-segment we continue reading from the column immediately to the right of the last node of the previous $p$-segment. 
The quantity $j(\lambda)$ is defined as the number of special nodes in the $p$-rim of $\lambda$ that lie at a rightmost end of a row but are not the last node of a $p$-segment.

Example 5.4. Let $\lambda=(4,3,3,2,2,1)$ and $p=3$. The $p$-rim of the diagram of $\lambda$ consists of positions that are circled below.

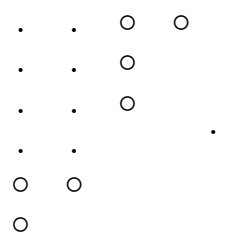

In this case $j(\lambda)=4$ and the special nodes are marked by $\bullet$ below

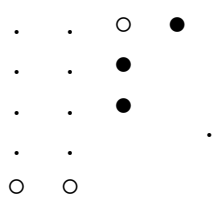

Theorem 5.5. The complete set of highest weights of simple $S(m \mid n, r)$ modules $L(\lambda)$ consists of partitions $\lambda=\left(\lambda_{1} \geq \ldots \geq \lambda_{m}, \lambda_{m+1} \geq \ldots \lambda_{m+n}\right)$ such that $j\left(\lambda_{m+1}, \ldots, \lambda_{m+n}\right) \leq \lambda_{m}$.

The proof of this theorem uses the method of odd reflections developed by Vera Serganova. She investigates how the highest vectors of simple modules are changing when one replaces a Borel supergroup by a different (non-conjugated) one which corresponds to replacement of one odd root by another.

\section{Quantum groups and quantum bideterminants}

We start with the definition of the quantum Schur algebra $S_{q}(n, r)$ and quantum general linear group $G L_{q}(n)$.

Let $A_{q}(n)$ be a $K$-algebra generated by noncommuting variables $c_{i j}$ for $1 \leq i, j \leq n$ subject to relations

$$
\begin{array}{ll}
c_{i l} c_{i k}=q c_{i k} c_{i l} & 1 \leq k<l \leq n \\
c_{j k} c_{i k}=q c_{i k} c_{j k} & 1 \leq i<j \leq n \\
c_{i l} c_{j k}=c_{j k} c_{i l} & 1 \leq i<j, k<l \leq n \\
c_{i k} c_{j l}-c_{j l} c_{i k}=\left(q^{-1}-q\right) c_{i l} c_{j k} & 1 \leq i<j, k<l \leq n
\end{array}
$$

where $q$ is the quantum variable. 
Then $A_{q}(n)$ is a bialgebra with respect to the comultiplication $\Delta\left(c_{i j}\right)=\sum_{1 \leq k \leq n} c_{i k} \otimes c_{k j}$ and counit $\epsilon\left(c_{i j}\right)=\delta_{i j}$. The bialgebra $A_{q}(n)=\oplus_{r \geq 0} A_{q}(n, r)$ has an obvious grading by degree, where each homogeneous component $A_{q}(n, r)$ is a supercoalgebra. Its dual $S_{q}(n, r)=$ $A_{q}(n, r)^{*}$ is a quantum Schur algebra, or $q$-Schur algebra for short.

The $q$-Schur algebras were introduced by Dipper and James [14] as endomorphism modules of certain modules over Hecke algebras. For a nice introduction to $q$-Schur algebras we refer the reader to a manuscript of Mathas [34]. The approach to quantum group we use is due to Parshall and Wang [36] and Dipper and Donkin [13]. (See also Martin [33] and Donkin [17]).

Denote by $\ell(\sigma)$ the length of permutation $\sigma \Sigma_{r}$.

The quantum determinant $D$ is defined as

$$
D=\sum_{\sigma \in \Sigma_{n}}(-q)^{-\ell(\sigma)} c_{\sigma(1), 1} c_{\sigma(2), 2} \ldots c_{\sigma(n), n} .
$$

The coordinate algebra $\mathcal{O}\left(G L_{q}(n)\right)$ of the quantum linear group $G L_{q}(n)$ is defined as the Ore localization of $A_{q}(n)$ with respect to the multiplicative set $\left\{D^{r} \mid r \geq 0\right\}$.

If $T_{i}, T_{j}$ are tableaux of shape $\lambda$, where entries in $T_{i}$ are column increasing, then the $q$-bideterminant $\left(T_{i}: T_{j}\right)$ is defined as

$$
\left(T_{i}: T_{j}\right)=\sum_{\sigma \in C(T)}(-q)^{-\ell(\sigma)} c_{i \sigma, j}
$$

The following theorem is due to [24]. (See also Proposition 1.3.4 of [17].)

Theorem 6.1. The set $\left(T_{i}: T_{j}\right)$, where $T_{i}, T_{j}$ are semistandard tableaux of shape $\lambda$ for any partition $\lambda$ of $r$ is a basis of $A_{q}(n, r)$.

Define $D_{q}(\lambda)$ to be the vector space spanned by all bideterminants $\left(T_{\ell}\right.$ : $\left.T_{i}\right)$ of shape $\lambda$. Then $D_{q}(\lambda)$ is a $S_{q}(n, r)$-module, called the $q$-Schur module. The following theorem follows from [23]. (See also Proposition 4.5.2 of [17].)

Theorem 6.2. The module $D_{q}(\lambda)$ has a basis consisting of bideterminants $\left(T_{\ell}: T_{i}\right)$, where $T_{i}$ is a standard tableaux of shape $\lambda$.

As in the classical case, the module $D_{q}(\lambda)$ has a simple socle $L_{q}(\lambda)$.

Theorem 6.3. The set of all non-isomorphic simple $G L_{q}(n)$-modules is parametrized by the set of all partitions $\lambda$ and consists of modules $L_{q}(\lambda)$. $[17])$.

Finally, we conclude with the following theorem due to [36]. (See also 
Theorem 6.4. The algebra $S_{q}(n, r)$ is quasi-hereditary.

Another proof of this theorem is given in [34] using a basis due to G.E. Murphy.

\section{References}

[1] M.Barnabei, Schur modules, Weyl modules, and Capelli operators, Adv. in Math. 151 (2000), 1-35.

[2] A.Berele and A.Regev, Hook Young diagrams with applications to combinatorics and to representations of Lie superalgebras, Adv. Math., 64 (1987), 118-175.

[3] A. Brini, Combinatorics, superalgebras, invariant theory and representation theory, Sem. Lothar. de Comb. 55 (2007), Article B55g.

[4] J.Brundan and J.Kujawa, A new proof of the Mullineux conjecture, J. Algebraic. Combin., 18 (2003), no.1, 13-39.

[5] R.W.Carter and G.Lusztig, On the modular representations of the general linear and symmetric groups, Math. Z. 136 (1974), 193-242.

[6] G. Cliff, A basis of bideterminants for the coordinate ring of the orthogonal group, Communications in Algebra 36 (2008), 2719-2749.

[7] E.Cline, B.Parshall and L.Scott, Finite dimensional algebras and highest weight categories, J. reine angew. Math., 391 (1988),85-99.

[8] C. De Concini, Symplectic standard tableaux, Adv. Math. 34 (1979) 1-27.

[9] C. De Concini and C. Procesi, A characteristic free approach to invariant theory, Adv. in Math. 21 (1976), 330-354.

[10] C. De Concini, D. Eisenbud and C. Procesi, Y oung diagrams and determinantal varieties, Invent. Math. 56 (1980), no. 2, 129-165.

[11] J. Desarmenien, An algorithm for the Rota straigtening formula, Discrete Math. 30 (1980), 51-68.

[12] J. Desarmenien, J.P.S.Kung and G.C. Rota, Invariant theory, Young bitableaux, and combinatorics, Adv. in Math. 27 (1978), 63-92.

[13] R. Dipper and S. Donkin, Quantum $G L_{n}$, Proc. London Math. Soc. (3) 63 (1991), 165-211.

[14] R. Dipper and G.D. James, The q-Schur algebras, Proc. London Math. Soc. 59 (1989), 23-50.

[15] S. Donkin, A filtration of rational G-modules, Math. Z. 177, 1-8.

[16] S. Donkin, Representations of symplectic groups and the symplectic tableaux of R.C. King, Linear and Multilinear Algebra 29 (1991) 113-124.

[17] S. Donkin, The q-Schur algebra, London Math. Soc. Lecture Note Series 253, Cambridde University Press 1998.

[18] P. Doubilet, G. C. Rota, and J. Stein, On The Foundations of Combinatorial Theory, Vol. IX, Studies in Applied Mathematics, Vol. 53, (1974), 185-216.

[19] J.A.Green, Polynomial representations of $\mathrm{GL}_{n}$, Lecture Notes in Math., 830, Springer-Verlag, 1980.

[20] J.A.Green, Combinatorics and the Schur algebra, J. Pure Applied Algebra, 88 (1993), 89-106.

[21] F.D.Grosshans, G.C.Rota and J.A.Stein, Invariant theory and superalgebras, Regional Conference Series in Mathematics, Vol. 69 Amer.Math. Soc., Providence RI, 1987.

[22] W. V. D. Hodge, Some enumerative results in the theory of forms, Proc. Cambridge Philos. Soc. 39 (1943), 22-30. 
[23] J. Hu, A combinatorial approach to representations of quantum linear groups, Comm. Algebra 26 (1998), 2591-2621.

[24] R.Q. Huang and J.J. Zhang, Standard basis theorem for quantum linear groups, Adv. Math 102 (1993), 202-229.

[25] J. Igusa, On the arithmetic normality of the Grassmann variety, Proc. Nat. Acad. Sc. U.S.A. 40 (1954), 309-313.

[26] G.D.James, The Representation Theory of the Symmetric Groups, Lecture Notes in Math., 682, Springer-Verlag, 1978.

[27] G.D.James, On the decomposition matrices of the symmetric gropus III, J. Algebra 71, 115-122.

[28] G.D.James and A.Kerber, The Representation Theory of the Symmetric Group, Encyclopedia of Mathematics and its Applications 16, Addison-Wesley, Reading 1981.

[29] R. King, Weight multiplicities for classical groups, in: Group Theoretical Methods in Physics (Fourth International Colloquium, Nijmegen 1975), in: Lecture Notes in Phys., vol. 50, Springer, Berlin/Heidelberg/New York, 1975.

[30] M. Maliakas, On polynomial representations and filtrations for the special orthogonal groups, J. Algebra 168 (1994), no. 1, 143-171.

[31] F.Marko and A.N.Zubkov, Schur superalgebras in characteristic p, II, Bull. London Math. Soc. 38 (2006), 99-112.

[32] F.Marko and A.N.Zubkov, A note on bideterminants for Schur superalgebras, J. Pure Appl. Algebra 215 (2011) (9), 2223-2230.

[33] S. Martin, Schur algebras and representation theory, Cambridge Tracts in Mathematics, 112, Cambridge University Press, Cambridge, 1993.

[34] A. Mathas, Iwahori-Hecke algebras and Schur algebras of the symmetric group, Univ. Lecture Notes, 15, Amer. Math. Soc., Providence, 1999.

[35] N.J.Muir, Polynomial representations of the general linear Lie superalgebra, Ph.D. Thesis, University of London, 1991.

[36] B. Parshall and J.P. Wang, Quantum linear groups, Memoirs Amer. Math. Soc. 439, Amer. Math. Soc. Providence 1991.

[37] B.E. Sagan, The symmetric group: Representations, combinatorial algortithms, and symmetric functions, Graduate Texts in Mathematics 203, Springer-Verlag, New York, 2001.

[38] A.N.Sergeev, Tensor algebra of the identity representation as a module over the Lie superalgebras $\mathrm{GL}(n, m)$ and $\mathrm{Q}(n)$, Mat. Sb. (N.S.) 123 (165) (1984) 422-430 (in Russian), Math. USSR Sbornik 51 (1985) 419-427 (English translation).

[39] A. Stokke, A symplectic Desarmenien matrix and a basis for the symplecticWeyl module, J. Algebra 272 (2) (2004), 512-529.

[40] M.H.Peel, Specht modules and symmetric groups, J. of Algebra, 36 (1975), 88-97.

[41] B.W.Wetherilt, A note on amodule in tensor space for the symplectic group, J. Algebra 103 (1986) 294-301.

[42] A. Young, On quantitative substitutional analysis I, Proc. London Math. Soc. 33 (1901), 97-146.

[43] A. Young, On quantitative substitutional analysis III, Proc. London Math. Soc. (2) $\mathbf{2 8}$ (1927), 255-292.

[44] A.N. Zubkov, Some properties of general linear supergroups and of Schur superalgebras, Algebra and Logic 45 (2006) (3), 147-171.

São Paulo J.Math.Sci. 6, 1 (2012), 81-96 